\title{
Prognostic value of inflammatory markers in patients with COVID-19 in Indonesia
}

\author{
Ignatius Bima Prasetya ${ }^{\mathrm{a}, 1}$, Cucunawangsih ${ }^{\mathrm{b}}$, Jane Olivia Lorens ${ }^{\mathrm{a}}$, Veli Sungono ${ }^{\mathrm{b}}$, \\ Korri Elvanita El-Khobar ${ }^{c}$, Ratna Sari Wijaya ${ }^{\text {b, } *, 1}$ \\ ${ }^{a}$ Department of Internal Medicine, Faculty of Medicine, Pelita Harapan University, Tangerang, Indonesia \\ ${ }^{\mathrm{b}}$ Department of Microbiology, Faculty of Medicine, Pelita Harapan University, Tangerang, Indonesia \\ ${ }^{\text {c }}$ Eijkman Institute for Molecular Biology, Jakarta, Indonesia
}

\section{A R T I C L E I N F O}

\section{Keywords:}

COVID-19

C-reactive protein

Neutrophil to lymphocyte ratio

Risk factors

\begin{abstract}
A B S T R A C T
Background \& aims: Coronavirus disease 2019 (COVID-19) has a wide clinical spectrum, ranging from asymptomatic infection to severe diseases with high mortality rate. Early identification of high-risk COVID-19 patients may be beneficial to reduce morbidity and in-hospital mortality. This study aimed to investigate whether baseline levels of inflammatory markers such as C-reactive protein (CRP) and immune-cell-based inflammatory indices, neutrophil to lymphocyte ratio (NLR), derived-NLR (d-NLR), and platelet to lymphocyte ratio (PLR) at hospital admission are associated with adverse disease outcomes in COVID-19 patients.

Methods: Clinical data from 391 hospitalized COVID-19 patients in three Siloam Hospitals in Indonesia were retrospectively collected and analysed from March 20 to October 30, 2020.

Results: Fifty-four (13.8\%) hospitalized patients had clinical deterioration and required ICU treatment, categorizing them as severe COVID-19 cases. Older age, presence of underlying diseases, and increased inflammatory markers values at admission were significantly associated with severe cases. After adjustment of sociodemographic and comorbidities factors, CRP, NLR, and d-NLR values, but not PLRs, were identified as independent risk factors for disease severity and death in COVID-19 patients. The area under curve (AUC) of CRP, NLR, and dNLR were $0.854,0.848$, and 0.854 , respectively. The optimal cut-off points for CRP, NLR, and d-NLR for identification of COVID-19 patients with potential worse disease outcomes were $47 \mathrm{mg} / \mathrm{L}, 6$, and 4, respectively.

Conclusion: Initial assessment of CRP, NLR, and d-NLR values at hospital admission may be important predictors for adverse disease outcomes in COVID-19 patients.
\end{abstract}

\section{Introduction}

A novel human coronavirus, severe acute respiratory syndrome coronavirus 2 (SARS-CoV-2), causing the COVID-19 diseases has spread globally. Indonesia reported its first cases on March 2, 2020, and currently, there were more than 1 million cases of COVID-19 as of February $2,021 .^{1}$ The case fatality rate (CFR) for COVID-19 in Indonesia is higher than worldwide $(2.7 \%$ vs $2.3 \%) .^{2}$ COVID-19 infection has broad clinical spectrum of diseases, ranging from asymptomatic infection to life-threatening illnesses. ${ }^{3}$ In patients with severe infection, the disease might rapidly progress into acute respiratory distress syndrome (ARDS), coagulopathy, septic shock, and even death. ${ }^{3}$ Early identification of high-risk COVID-19 patients is necessary to prompt suitable medical intervention to reduce morbidity and in-hospital mortality. However, due to the large numbers of daily COVID-19 cases increase and relatively insufficient medical resources in Indonesia, ${ }^{1,4}$ the screening of such high-risk patients becomes a challenge.

Recent studies have shown that inflammatory markers like C-reactive protein (CRP), and immune-cell-based inflammatory indices such as neutrophil to lymphocyte ratio (NLR), derived neutrophil to lymphocyte ratio (d-NLR), and platelet to lymphocyte ratio (PLR), were significantly associated with adverse disease outcomes of COVID-19 and can be utilized to identify high-risk COVID-19 patients at early stage.$^{5-7}$ However, the optimum cut-off for these inflammatory markers was widely varying across studies. There was no universal cut-off threshold for these parameters to predict COVID-19 patients with potentially have clinical

\footnotetext{
* Corresponding author. Faculty of medicine, University of Pelita Harapan, MH Thamrin Boulevard, 1100, Banten, Indonesia.

E-mail address: ratna.wijaya@uph.edu (R.S. Wijaya).

1 These authors equally contributed.
} 
deterioration. Therefore, in this study, we aim to explore whether the baseline inflammation markers values of CRP, NLR, d-NLR, and PLR at hospital admission are associated with an increased risk of disease severity and death in hospitalized COVID-19 patients and to identify the best cut-off of these inflammatory markers that can be used in the early stratification risk of COVID-19 disease.

\section{Materials and methods}

\subsection{Study design and participants}

This study was a retrospective observational study on COVID-19 patients who were hospitalized in three Siloam Hospitals located in Jakarta, Banten, and West Java provinces of Indonesia from 20 March 2020 to 30 October 2020. This study was approved by the research ethics committee of Faculty Medicine of Pelita Harapan University (No: 147/K-LKJ/ETIK/VI/2020) and has been granted with waiver of collecting informed consent from study participants.

COVID-19 diagnosis was based on positive SARS-CoV-2 RNA detection in nasopharyngeal swab specimens by reverse-transcriptase polymerase chain reaction (RT-PCR). Exclusion criteria were age younger than 18 years, pregnant patients, patients with missing clinical and hematology data, and those without clinical outcomes data. The degree of severity of COVID-19 (non-severe versus severe) on admission was assessed using the latest guidelines for coronavirus disease diagnosis and treatment protocol from the Indonesia Ministry of Health. As outlined in the guidelines, patients were categorized into four types: mild, moderate, severe, and critical illness. Mild cases included patients with mild clinical symptoms and no signs of pneumonia in imaging or hypoxia. Moderate cases included patients with clinical signs of pneumonia (fever, cough, dyspnoea, fast breathing) but no signs of severe pneumonia, including $\mathrm{SpO}_{2} \geq 93 \%$. Severe cases included patients who met one of the following criteria: (1) respiratory rate $>30$ counts per minute, (2) $\mathrm{SpO}_{2}<93 \%$, (3) signs of severe respiratory distress. Critical illness cases included patients who met any of the following criteria: (1) respiratory failure requiring mechanical ventilation, (2) the presence of sepsis and shock sepsis. Only COVID-19 patients with mild and moderate cases classified as non-severe cases and treated in a regular isolation ward on hospital admission were included in this study. Since previous studies have described the median time for COVID-19 clinical deterioration was 9-11 days after onset illness, ${ }^{8-10}$ we excluded patients with early admission $(<48 \mathrm{~h})$ to ICU since they were more likely to already be in a severe or critically ill condition, which may confound the analyses results.

\subsection{Data collection and definitions}

A trained team of physicians reviewed and collected the epidemiological, clinical, laboratory, and clinical outcomes data from the electronic medical records. The laboratory parameters CRP, white blood cells (WBCs), neutrophil, lymphocyte, and platelet count were measured at admission and used to calculate several ratios indicative of inflammatory markers. The NLR was calculated by dividing the neutrophil count with lymphocyte count. The d-NLR was measured by dividing the neutrophil count with WBCs minus the neutrophil count, while the PLR was obtained by dividing the platelet count with lymphocyte count.

\subsection{Outcomes}

The primary outcome of this study was COVID-19 severity based on worse clinical progression experienced by each case during hospitalization. The endpoint for severe COVID-19 was defined as admission to ICU. The criteria for ICU admission were: (1) respiratory rate $>30$ counts per minute, (2) resting pulse oxygen saturation $<93 \%$, (3) signs of severe respiratory distress, and (4) respiratory failure and/or signs of shock. The secondary outcome was all-cause mortality within 30 days of hospitalized COVID-19 patients.

\subsection{Data analysis}

The Kolmogorov-Smirnov test was used to evaluate the normality distribution of quantitative data. Mean and standard deviation (SD) was used to describe normally distributed numeric variables, while median with the 25th-75th interquartile range (IQR) was used to describe nonnormally distributed numeric variables. Nominal variables were shown as counts and percentages. Independent group $t$-test was used for normally distributed numeric variables and Mann-Whitney $U$ test was used for non-normally distributed numeric variables. $\chi^{2}$ test or Fisher's exact test was used for nominal variables. Univariate and multivariate logistic regression models were used to examine the risk factors of disease severity. We selected variables from demographics and comorbidities for inclusion in the multivariate logistic regression based on statistical reasoning from the univariate results $(\mathrm{p}<0.25)$. The variance inflation factor (VIF) indicator was assessed to investigate the amount of multicollinearity within variables in the multivariate regression model. Receiver operating characteristic (ROC) curve analysis was performed to assess the predictive value of various inflammatory markers on admission for predicting COVID-19 severity. The sensitivity and specificity with $95 \%$ confidence intervals (CIs), as well as the optimal cut-off value for each predictor based on the maximum value of the Youden's index were measured. The areas under the curve (AUC) were calculated using DeLong's method. We used Graphpad Prism (version 9.0.1), R software (version 3.6.3), and easyROC (version 1.3.1) for statistical analysis and graph drawing. In all analyses, a two-tailed p-value of less than 0.05 was considered as statistically significant.

\section{Results}

\subsection{Characteristics of study subjects}

During the study period (from 20 March 2020 to 30 October 2020), 731 patients with confirmed COVID-19 diagnosis were admitted to Siloam Hospitals. Only 391 patients were included in the final analysis (Fig. 1). Patients who were admitted to ICU on the first day of admission $(\mathrm{n}=15)$, patients without available baseline clinical and laboratory results $(n=259)$, patients who were pregnant $(n=5)$, patients under 18 years $(n=6)$, and patients who were transferred to other hospitals ( $n=$ 55) were excluded from the analysis.

The baseline clinical characteristics of COVID-19 patients in our study were summarized in Table 1 . The median age was 43 years (IQR $32-54$ ) and $62 \%$ were male. The median time from symptom onset to admission was seven days (IQR 5-7). The most prevalent underlying medical conditions were hypertension $(17.7 \%)$ and diabetes $(11.3 \%)$. Cough (59.6\%), fever (56.8\%), and dyspnoea (35.5\%) were the most

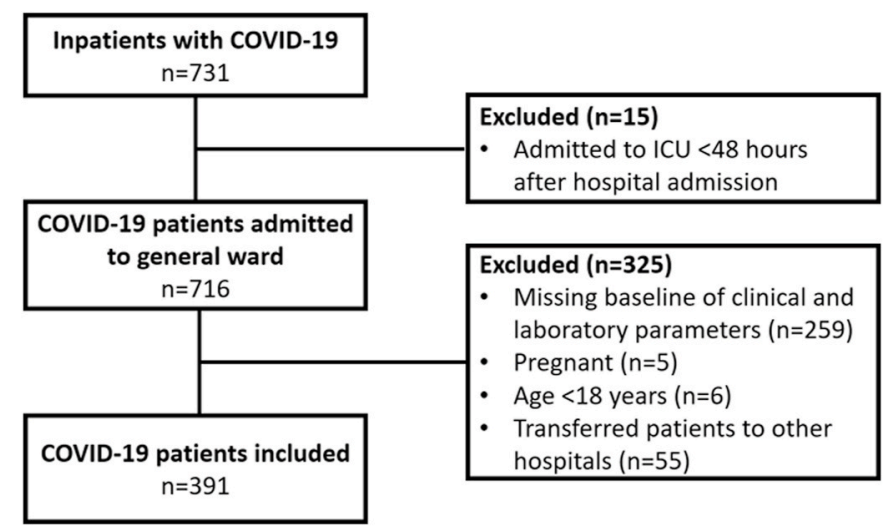

Fig. 1. Flow chart of patient inclusion. 
Table 1

Demographic, clinical characteristics, and clinical outcomes of COVID-19 patients.

\begin{tabular}{ll}
\hline Variables & Total $(\mathrm{n}=391)$ \\
\hline Demographics & \\
Age (years) & $43(32-54)$ \\
Gender (male) & $243(62)$ \\
Time from symptoms to hospitalization (days) & $7(5-7)$ \\
Comorbidities & \\
Diabetes & $44(11.3)$ \\
Hypertension & $69(17.7)$ \\
Chronic heart diseases & $16(4.1)$ \\
Chronic lung diseases & $24(6.1)$ \\
Symptoms & \\
Fever & $222(56.8)$ \\
Fatigue & $77(19.7)$ \\
Cough & $233(59.6)$ \\
Anorexia & $66(16.9)$ \\
Anosmia & $35(9)$ \\
Myalgia & $49(12.5)$ \\
Dyspnoea & $139(35.5)$ \\
Sore throat & $61(15.6)$ \\
Diarrhoea & $44(11.3)$ \\
Abdominal pain & $45(11.5)$ \\
Nausea & $93(23.8)$ \\
Vomiting & $24(6.1)$ \\
Headache & $60(15.3)$ \\
Chest CT images, & \\
Ground-glass opacities & $218(65.1)$ \\
Pulmonary fibrosis & $3(0.9)$ \\
Bronchiectasis & $3(0.9)$ \\
Tuberculosis & $4(1.2)$ \\
Clinical outcomes & \\
ICU admission & $54(13.8)$ \\
Death & $22(5.6)$ \\
\hline Datare presented & \\
\hline & \\
\hline & \\
\hline
\end{tabular}

Data are presented as median (interquartile range, IQR) or count (\%).

Abbreviations: CT, computed tomography; ICU, intensive care unit.

common symptoms on hospital admission. Of the 335 patients with chest CT scan on admission, most 218 (65.1\%) had abnormal results, showing typical images of ground-glass opacities (Table 1). Out of 391 hospitalized COVID-19 patients, 54 (13.8\%) patients had clinical deterioration and required treatment in the ICU (severe cases) and $22(5.6 \%)$ patients died during hospitalization. The median time between hospital admission to ICU admission was 4 (3-5) days.

The results of the peripheral blood of the COVID-19 patients on the day of hospital admission were listed in Table 2. COVID-19 patients who had disease progression and required ICU treatment showed significantly higher baseline levels of WBCs (11.9 vs. $7.5 \times 10^{3}$ cells $/ \mu \mathrm{L} ; \mathrm{p}<$ 0.001 ), neutrophil count ( 9.3 vs. $4.7 \times 10^{3}$ cells $\left./ \mu \mathrm{L} ; \mathrm{p}<0.001\right)$, but lower levels of platelets ( 242.5 vs. $289 \times 10^{3}$ cells $\left./ \mu \mathrm{L} ; \mathrm{p}=0.001\right)$ and lymphocytes ( 0.9 vs. $1.8 \times 10^{3}$ cells $/ \mu \mathrm{L} ; \mathrm{p}<0.001$ ). Patient with severe COVID-19 as defined by ICU admission had a significant elevation baseline level of inflammatory markers CRP, NLR, d-NLR, and PLR ( $\mathrm{p}<$ 0.001 , respectively). In addition, compared to patients who survived and discharged from the hospital (survivor), the significant elevation of WBCs, neutrophil count, and inflammatory markers; CRP, NLR, d-NLR, PLR, as well as the lower platelets and lymphocytes were observed on patients who died during hospitalization (non-survivor).

\subsection{Association of baseline inflammatory markers values with COVID-19 disease severity and mortality}

Based on the univariate logistic regression analysis, patients' inflammatory markers values at admission were associated with disease severity of COVID-19 (Table 3). The confounding variables with an increased risk of severe COVID-19 were age (OR 1.1; $\mathrm{p}<0.001$ ), diabetes (OR 8.7; $\mathrm{p}<0.001$ ), hypertension (OR 4.2; $\mathrm{p}<0.001$ ), and chronic heart disease (OR 5.4; $\mathrm{p}<0.001$ ) (Supplementary Table 1). Table 3 showed that even after adjustment of age, gender, and relevant comorbidities, the values of CRP, NLR, and d-RNL at admission remained significantly associated with a higher risk of severe disease. The elevated baseline CRP values were associated with a 1.02-fold higher risk of severe disease (OR 1.02; 95\% CI 1.02-1.03). The higher baseline of NLR levels was associated with a 1.25-fold higher risk of severe disease (OR 1.25; 95\% CI 1.16-1.36). The odd ratios of severe disease increased by 1.68 -fold for any increment of d-NLR levels on admission (OR 1.68; 95\% CI 1.44-2.01). PLR was related with severe outcome in the univariable analysis but did not reach statistical significance after factors adjustment $(\mathrm{p}=0.05)$.

Furthermore, the univariate logistic regression analysis results showed age, diabetes, hypertension, chronic heart disease, and chronic lung disease significantly associated with a risk increase of COVID-19 mortality (Supplementary Table 2). As showed in Table 3, the crude OR for COVID-19 mortality was 1.02 (95\% CI 1.01-1.02) for the elevation of CRP values on admission, 1.17 (95\% CI 1.11-1.23) for baseline NLR elevation, and 1.41 (95\% CI 1.26-1.6) for baseline d-NLR elevation. Table 3 showed that after adjustment confounding variables, elevation of the CRP, NLR, and d-NLR at admission was significantly correlated with a higher risk of COVID-19 mortality. Elevation of CRP values was associated with a 1.01-fold risk increase of death. The increased NLR parameter value on admission was associated with a 1.12-fold risk increased for COVID-19-related death, while baseline dNLR elevation was related with a 1.33-fold higher risk of COVID-19 mortality.

Table 2

Laboratory findings of COVID-19 patients.

\begin{tabular}{|c|c|c|c|c|c|c|c|}
\hline \multirow[t]{2}{*}{ Variables } & \multirow[t]{2}{*}{ Normal range } & \multicolumn{3}{|l|}{ Primary outcome } & \multicolumn{3}{|l|}{ Secondary outcome } \\
\hline & & non-ICU (n = 337) & ICU $(n=54)$ & p-value & Survivor $(\mathrm{n}=369$ ) & Non-survivor $(\mathrm{n}=22)$ & p-value \\
\hline WBCs, $10^{3}$ cells $/ \mu \mathrm{L}$ & $4-10$ & $7.5(5.5-9.3)$ & $11.9(8-17.4)$ & $<0.001$ & $7.6(5.6-9.7)$ & $13.8(8.5-18.8)$ & $<0.001$ \\
\hline Neutrophil, $10^{3}$ cells $/ \mu \mathrm{L}$ & $2-7$ & $4.7(3.2-6.5)$ & $9.3(6.4-14.4)$ & $<0.001$ & $4.9(3.3-6.9)$ & $11.7(7-14.6)$ & $<0.001$ \\
\hline Lymphocyte, $10^{3}$ cells $/ \mu \mathrm{L}$ & $0.8-4$ & $1.8(1.3-2.3)$ & $0.9(0.7-1.4)$ & $<0.001$ & $1.7(1.2-2.2)$ & $0.8(0.6-1.1)$ & $<0.001$ \\
\hline Platelet, $10^{3}$ cells $/ \mu \mathrm{L}$ & $150-400$ & $289(231-341)$ & $242.5(166.8-340)$ & 0.001 & $288(231-340.5)$ & $207(128.8-313)$ & 0.001 \\
\hline CRP, mg/L & $<10$ & $4(1-16.5)$ & 86.5 (12.5-169.8) & $<0.001$ & $4(1-20.8)$ & $74.4(24.7-201.8)$ & $<0.001$ \\
\hline NLR & & $2(2-4)$ & $9.5(5.8-18)$ & $<0.001$ & $2.6(1.9-4.3)$ & $14.6(6.6-22.5)$ & $<0.001$ \\
\hline d-NLR & & $2(1-3)$ & $6(4-10)$ & $<0.001$ & $1.9(1.4-2.9)$ & $7(4.5-10.1)$ & $<0.001$ \\
\hline PLR & & 160 (123.5-209) & 254 (160.5-383.5) & $<0.001$ & $164.4(127-224.5)$ & $199.6(154.1-443.5)$ & $<0.001$ \\
\hline
\end{tabular}

Data are presented as median (interquartile range, IQR).

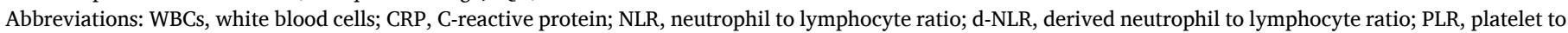
lymphocyte ratio. 
Table 3

Risk factors associated with severity and mortality of COVID-19 infection.

\begin{tabular}{|c|c|c|c|c|c|c|c|c|}
\hline \multirow[t]{2}{*}{ Variables } & \multirow[b]{2}{*}{ Crude OR $(95 \% \mathrm{CI})$} & \multicolumn{2}{|c|}{ ICU-admission } & \multirow[b]{2}{*}{ p-value } & \multirow[b]{2}{*}{ Crude OR $(95 \%$ CI $)$} & \multicolumn{2}{|c|}{ COVID-19 mortality } & \multirow[b]{2}{*}{ p-value } \\
\hline & & p-value & Adjusted $^{\mathrm{a}}$ OR $(95 \% \mathrm{CI})$ & & & $\mathrm{p}$-value & Adjusted $^{\mathrm{a}}$ OR (95\% CI) & \\
\hline CRP & $1.026(1.020-1.033)$ & $<0.001$ & $1.021(1.015-1.029)$ & $<0.001$ & $1.015(1.010-1.020)$ & $<0.001$ & $1.011(1.005-1.017)$ & $<0.001$ \\
\hline NLR & 1.295 (1.213-1.397) & $<0.001$ & 1.250 (1.164-1.359) & $<0.001$ & $1.166(1.109-1.231)$ & $<0.001$ & $1.124(1.065-1.192)$ & $<0.001$ \\
\hline d-NLR & $1.806(1.569-2.120)$ & $<0.001$ & 1.679 (1.439-2.008) & $<0.001$ & 1.409 (1.259-1.599) & $<0.001$ & $1.325(1.185-1.504)$ & $<0.001$ \\
\hline PLR & $1.000(1.001-1.004)$ & 0.001 & $1.002(1.000-1.003)$ & 0.050 & $1.003(1.001-1.005)$ & 0.001 & $1.001(1.000-1.003)$ & 0.050 \\
\hline
\end{tabular}

Abbreviations: CI, confidence interval; OR, odds ratio, CRP, C-reactive protein; NLR, neutrophil to lymphocyte ratio; d-NLR, derived neutrophil to lymphocyte ratio; PLR, platelet to lymphocyte ratio.

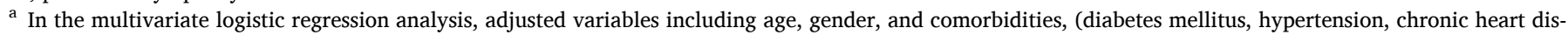
eases, and chronic lung diseases).

\subsection{ROC analysis of inflammatory markers}

To investigate the accuracy of each inflammatory markers and their best cut-off value in predicting severe disease, ROC analysis was performed. Fig. 2 displayed the ROC curves of CRP, NLR, d-NLR, and PLR inflammatory markers in predicting worse disease progression in COVID-19 patients.
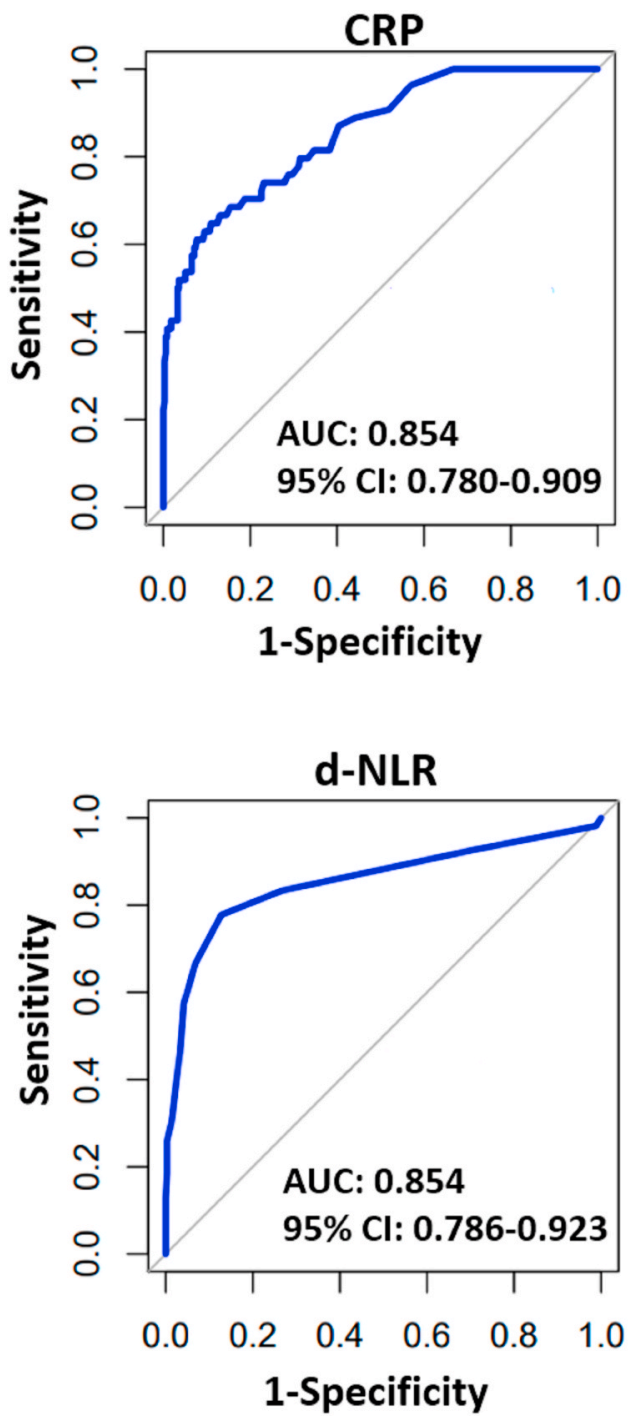

The AUC of CRP, NLR, d-NLR, and PLR in predicting severe COVID19 disease was 0.854 (95\% CI $0.780-0.909), 0.848$ (95\% CI 0.777-0.919), 0.854 (95\% CI 0.786-0.923), and 0.699 (95\% CI 0.615-0.783), respectively. There were no significant differences in AUC among CRP, NLR, and d-NLR, indicating comparable performance of these inflammatory markers to predict disease severity (Supplementary Table 3). In contrast, PLR performance to predict disease severity of
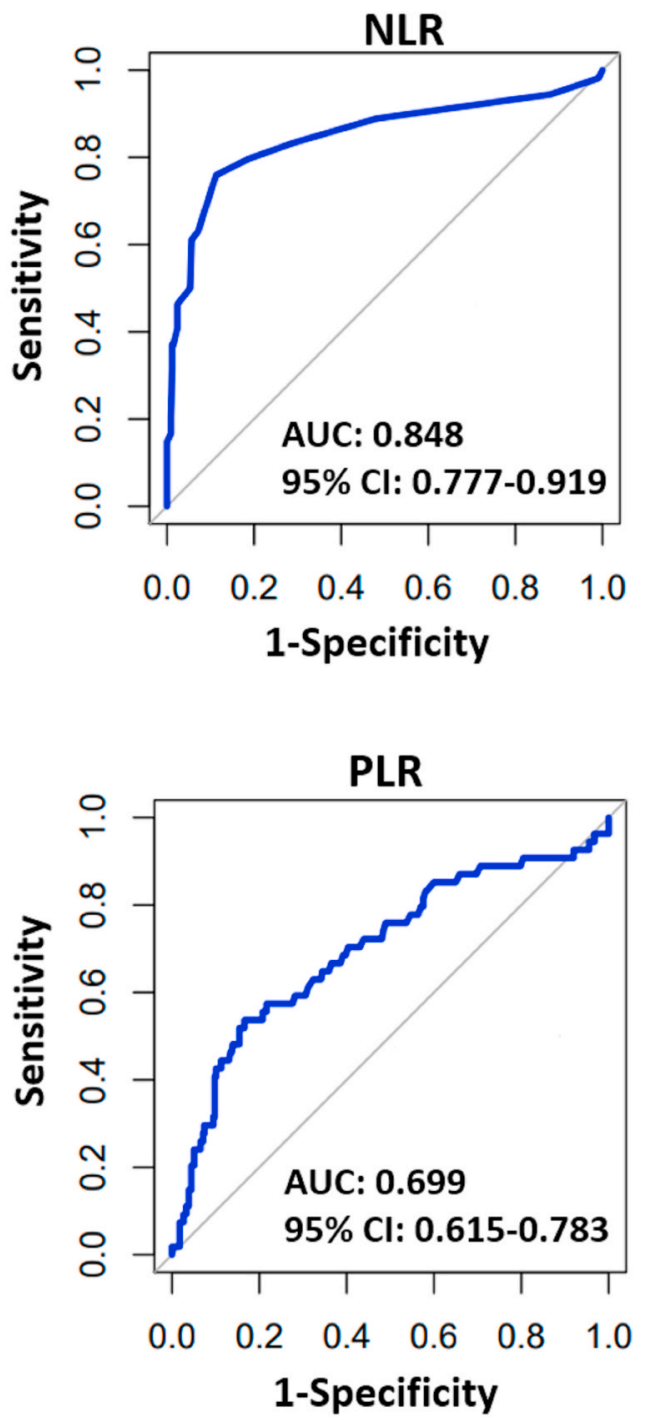

Fig. 2. Receiver Operator Characteristic (ROC) curves of inflammatory markers for prediction of severe COVID-19.

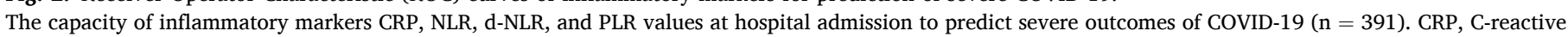

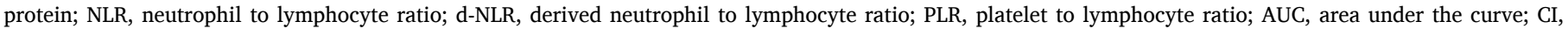
confidence interval. 
COVID-19 was significantly lower compared to CRP ( $p=0.005)$, NLR ( $p$ $=0.008)$, and d-NLR $(p=0.005)$. Based on the maximum value of the Youden index, CRP value at admission showed the best differentiation at $47 \mathrm{mg} / \mathrm{L}$ with $64.8 \%$ sensitivity and $89 \%$ specificity for predicting disease severity. The optimal cut-off points at admission to predict illness severity was 6 for NLR and 4 for d-NLR (Table 4).

\section{Discussion}

In this study, we provided potential markers for early risk assessment of COVID-19 patients at hospital admission using laboratory parameters. Although most COVID-19 patients experience only mild to moderate symptoms and have good disease prognosis, some COVID-19 patients can develop severe disease with increased mortality rate, varying from $30 \%$ to $60 \% .^{10-12}$ Our study found that values of CRP, NLR, or d-NLR inflammatory markers at hospital admission were an independent risk factor for poor disease outcomes in hospitalized COVID-19 patients.

We reported up to $14 \%$ of our hospitalized COVID-19 patients had worse disease progression and required treatment in the ICU. In agreement with other studies, ${ }^{13,14}$ patient's age was significantly associated with adverse outcomes of COVID-19 infection. This is believed to be related to immune senescence of the immune system and comorbidities that were more likely present in elderly patients. ${ }^{15,16}$ Additionally, the older age patients had higher expression of angiotensin-converting enzyme (ACE)-2, an entry receptor of SARS-CoV-2, in the alveolar region, and they had a lower frequency of lung progenitor cells for the lung repair process, potentially contributing to the severe manifestation of disease in older age. ${ }^{17}$ Hypertension was the most common underlying disease and had been significantly associated with disease severity and mortality, as reported previously. ${ }^{18,19}$ Although the mechanisms behind the negative impact of hypertension on COVID-19 remain unclear, the increased expression of ACE-2 due to antihypertensive drugs or ACE-2 gene polymorphism may possibly play negative role in COVID-19 patient with hypertension. ${ }^{20,21}$

Inflammatory response plays a major role in the pathophysiology of COVID-19 22. The amount of pro-inflammatory cytokines released following host immune response against SARS-CoV-2 is associated with the disease severity. ${ }^{12,23}$ Overproduction of proinflammatory cytokines might lead to severe or critical manifestations and cause hematology parameters changes, characterized by increased C-reactive protein (CRP), lymphocytopenia, neutrophilia, and thrombocytopenia. ${ }^{24}$ Thus, measurement of inflammatory markers such as CRP or immune-cell-based inflammatory indices NRL, d-NRL, and PLR may reflect the degree of systemic inflammatory response occurring in COVID-19 patients and can be used for early risk assessment of COVID-19 patients.

CRP has been widely utilized as an acute marker for inflammation, and CRP elevation is highly associated with poor disease outcomes in COVID-19 patients. $^{25,26}$ Our study showed that baseline CRP value in severe patients' group was higher than the non-severe group, and this

Table 4

Performance of inflammatory markers at hospital admission to predict worse disease progression in COVID-19 patients.

\begin{tabular}{|c|c|c|c|c|c|}
\hline Variables & $\begin{array}{l}\text { Youden } \\
\text { index }\end{array}$ & $\begin{array}{l}\text { Cut-off } \\
\text { point }\end{array}$ & $\begin{array}{l}\text { Sensitivity, \% } \\
(95 \% \mathrm{CI})\end{array}$ & $\begin{array}{l}\text { Specificity, \% } \\
(95 \% \text { CI) }\end{array}$ & p-value \\
\hline CRP & 0.54 & $>47$ & $\begin{array}{l}64.8 \\
(50.6-77.3)\end{array}$ & $\begin{array}{l}89.0 \\
(85.2-92.2)\end{array}$ & $<0.001$ \\
\hline NLR & 0.65 & $>6$ & $\begin{array}{l}75.9 \\
(62.4-86.5)\end{array}$ & $\begin{array}{l}88.7 \\
(84.9-91.9)\end{array}$ & $<0.001$ \\
\hline d-NLR & 0.65 & $>4$ & $\begin{array}{l}77.8 \\
(64.4-88.0)\end{array}$ & $\begin{array}{l}87.2 \\
(83.2-90.6)\end{array}$ & $<0.001$ \\
\hline PLR & 0.37 & $>248$ & $\begin{array}{l}53.7 \\
(39.6-67.4)\end{array}$ & $\begin{array}{l}83.4 \\
(79.0-87.2)\end{array}$ & $<0.001$ \\
\hline
\end{tabular}

Abbreviations: CRP, C-reactive protein; NLR, neutrophil to lymphocyte ratio; $d-$ NLR, derived neutrophil to lymphocyte ratio; PLR, platelet to lymphocyte ratio. elevated CRP had significant association with disease severity and mortality in COVID-19 patients. The optimal cut-off point was $47 \mathrm{mg} / \mathrm{L}$ with AUC ranging between 0.8 and 0.9 , suggesting excellent accuracy of CRP as marker for predicting adverse disease outcomes in COVID-19 patients. Although many studies have shown that elevated CRP levels may increase risk of worse prognosis in COVID-19 patients, the cut-off point for the risk stratification was considerably varied ranging from 20.42 to $110 \mathrm{mg} / \mathrm{L} .{ }^{27,28}$ The high variability of CRP cut-off points across these studies may be due to several factors, for example, differences in the definition and identification of severe COVID-19 cases, differences in the studied population characteristics including age, gender, smoking status, hypertension, diabetes, and the presence of other conditions that potentially affect CRP concentration in blood including CRP or IL-6 gene polymorphism.

The immune-cell-based inflammatory indices reflect the alteration of immune cell numbers and distribution in COVID-19 infection. NLR is calculated by dividing the neutrophil to lymphocyte count. Similar with previous studies, ${ }^{29,30}$ we showed that NLR was an independent risk factor associated with disease severity and mortality in COVID-19 patients. The significant increase of NLR in severe COVID-19 patients can be explained by the prominent features of both neutrophilia $(>7000$ cells $/ \mu \mathrm{L})$ and lymphocytopenia $(<1000$ cells $/ \mu \mathrm{L})$ in these patients. The optimal threshold of NLR was 6 , with $75.9 \%$ sensitivity and $88.7 \%$ specificity to predict adverse outcomes in COVID-19 patients. This result was similar to previous large COVID-19 cohort study in China involving 12,862 individuals, which showed NLR value of 6 as the best predictive factor in discriminating patients with high or low risk of unfavourable disease outcomes. ${ }^{31}$

In line with prior studies, ${ }^{7,32}$ the significant association of PLR with COVID-19 disease severity and mortality was not observed even after adjustment of the sociodemographic characteristics and comorbid conditions in multivariate analysis. Additionally, PLR had lower performance than the other studied markers for predicting worse disease outcomes in COVID-19 disease, limiting its use in the clinical setting. The d-NLR performance for predicting adverse outcomes in COVID-19 patients was comparable with CRP and NLR. However, the d-NLR was associated with the highest risk of disease severity and death in COVID-19 patients. Altered turnover in all WBC lineages in COVID-19 disease has been shown previously. ${ }^{33}$ Since d-NLR calculation took account of all different types of leukocytes, this ratio may reflect both the host's immunity status and the degree of systemic inflammation within the host, which possibly explains its strong association with adverse disease outcomes in COVID-19 patients. In particular, the increased d-NLR values was more likely reflected the neutrophilia conditions of COVID-19 patients. Neutrophilia caused by the high levels of circulating pro-inflammatory cytokines had been shown related to poor outcomes of COVID-19 patients. $^{9}$ Moreover, the lung autopsies of deceased COVID-19 patients have revealed the presence of neutrophil infiltration in pulmonary capillaries, extravasation of neutrophil into alveolar space, and neutrophilic mucositis. ${ }^{34}$ The increased neutrophil frequency can be a source of the high level of reactive oxygen species (ROS) and excess neutrophil extracellular traps (NETs), resulting in a severe lung injury. ${ }^{34,35}$

There were several limitations in our study. Firstly, we performed a retrospective study and missing patients' data were unavoidable. Exclusion of patients with missing data also resulted in decrease statistical power. Secondly, the cut-off points for inflammatory markers in our findings needs to be validated in a larger prospective and multicentre cohort study.

In conclusion, our study has shown that increasing CRP, NLR, and dNLR at hospital admission might be considered as an important marker for early identification of COVID-19 patients with poor disease prognosis. Inclusion of these parameters in medical decision strategies could provide more effective and timely initiation of disease management to optimize the use of limited medical resources. 


\section{Declaration of competing interest}

The authors have declared no conflicts of interest.

\section{Acknowledgements}

The authors would like to thank all the medical staffs in Siloam Hospitals that were involved in data collection.

\section{Appendix A. Supplementary data}

Supplementary data to this article can be found online at https://doi. org/10.1016/j.cegh.2021.100803.

\section{Financial Support statement}

No external funding was received.

\section{Author contributions}

Designing research studies (IP, RW), acquiring data (IP, RW, JL), analyzing data (IP, RW), interpreting the results (IP, RW, CU, VS), and writing the manuscript (IP, RW, CU, KE).

\section{References}

1 Update on Coronavirus Disease in Indonesia. World Health Organization; 2021. htt ps://www.who.int/indonesia/news/novel-coronavirus. Accessed March 31, 2021

2 Situasi terkini perkembangan coronavirus diseases (COVID-19) Kementerian Kesehatan Republik Indonesia 2021. Feburary 28, 2021 https://infeksiemerging.kem kes.go.id/situasi-infeksi-emerging/situasi-terkini-perkembangan-coronavirus-disea se-covid-19-27-februari-2021.

3 Wu Z, McGoogan JM. Characteristics of and important lessons from the coronavirus disease 2019 (COVID-19) outbreak in China: summary of a report of 72314 cases from the Chinese center for disease control and prevention. J Am Med Assoc. 2020; 323(13):1239-1242.

4 Phua J, Faruq MO, Kulkarni AP, et al. Critical care bed capacity in Asian countries and regions. Crit Care Med. 2020;48(5):654-662.

5 Feng X, Li S, Sun Q, et al. Immune-inflammatory parameters in COVID-19 cases: a systematic review and meta-analysis. Front Med. 2020;7:301.

6 Chan AS, Rout A. Use of neutrophil-to-lymphocyte and platelet-to-lymphocyte ratios in COVID-19. J Clin Med Res. 2020;12(7):448-453.

7 Yang AP, Liu JP, Tao WQ, Li HM. The diagnostic and predictive role of NLR, d-NLR and PLR in COVID-19 patients. Int Immunopharm. 2020;84:106504.

8 Chen SL, Feng HY, Xu H, et al. Patterns of deterioration in moderate patients with COVID-19 from jan 2020 to mar 2020: a multi-center, retrospective cohort study in China. Front Med. 2020;7:567296.

9 Wang D, Hu B, Hu C, et al. Clinical characteristics of 138 hospitalized patients with 2019 novel coronavirus-infected pneumonia in Wuhan, China. J Am Med Assoc. 2020;323(11):1061-1069.

10 Yang X, Yu Y, Xu J, et al. Clinical course and outcomes of critically ill patients with SARS-CoV-2 pneumonia in Wuhan, China: a single-centered, retrospective, observational study. Lancet Respir Med. 2020;8(5):475-481.
11 Bhatraju PK, Ghassemieh BJ, Nichols M, et al. Covid-19 in critically ill patients in the seattle region - case series. N Engl J Med. 2020;382(21):2012-2022.

12 Huang C, Wang Y, Li X, et al. Clinical features of patients infected with 2019 novel coronavirus in Wuhan, China. Lancet. 2020;395(10223):497-506.

13 Liu Y, Mao B, Liang S, et al. Association between age and clinical characteristics and outcomes of COVID-19. Eur Respir J. 2020;55(5).

14 Grasselli G, Greco M, Zanella A, et al. Risk factors associated with mortality among patients with COVID-19 in intensive care units in lombardy, Italy. JAMA Intern Med. 2020;180(10):1345-1355.

15 Shaw AC, Goldstein DR, Montgomery RR. Age-dependent dysregulation of innate immunity. Nat Rev Immunol. 2013;13(12):875-887.

16 Divo MJ, Martinez CH, Mannino DM. Ageing and the epidemiology of multimorbidity. Eur Respir J. 2014;44(4):1055-1068.

17 Zhang Z, Guo L, Huang L, et al. Distinct disease severity between children and older adults with COVID-19: impacts of ACE2 expression, distribution, and lung progenitor cells. Clin Infect Dis. 2021;ciaa1911. https://doi.org/10.1093/cid/ciaa1911. In press.

$18 \mathrm{Li} \mathrm{X}, \mathrm{Xu} \mathrm{S}$, Yu M, et al. Risk factors for severity and mortality in adult COVID-19 inpatients in Wuhan. J Allergy Clin Immunol. 2020;146(1):110-118.

19 Gao C, Cai Y, Zhang K, et al. Association of hypertension and antihypertensive treatment with COVID-19 mortality: a retrospective observational study. Eur Heart J. 2020;41(22):2058-2066.

20 Ferrario CM, Jessup J, Chappell MC, et al. Effect of angiotensin-converting enzyme inhibition and angiotensin II receptor blockers on cardiac angiotensin-converting enzyme 2. Circulation. 2005;111(20):2605-2610.

21 Bosso M, Thanaraj TA, Abu-Farha M, Alanbaei M, Abubaker J, Al-Mulla F. The two faces of ACE2: the role of ACE2 receptor and its polymorphisms in hypertension and COVID-19. Mol Ther Methods Clin Dev. 2020;18:321-327.

22 Tay MZ, Poh CM, Renia L, MacAry PA, Ng LFP. The trinity of COVID-19: immunity, inflammation and intervention. Nat Rev Immunol. 2020;20(6):363-374.

23 Chen G, Wu D, Guo W, et al. Clinical and immunological features of severe and moderate coronavirus disease 2019. J Clin Invest. 2020;130(5):2620-2629.

24 Mangalmurti N, Hunter CA. Cytokine storms: understanding COVID-19. Immunity. 2020;53(1):19-25.

25 Liu F, Li L, Xu M, et al. Prognostic value of interleukin-6, C-reactive protein, and procalcitonin in patients with COVID-19. J Clin Virol. 2020;127:104370.

26 Wu C, Chen X, Cai Y, et al. Risk factors associated with acute respiratory distress syndrome and death in patients with coronavirus disease 2019 pneumonia in Wuhan, China. JAMA Intern Med. 2020;180(7):934-943.

27 Poggiali E, Zaino D, Immovilli P, et al. Lactate dehydrogenase and C-reactive protein as predictors of respiratory failure in CoVID-19 patients. Clin Chim Acta. 2020;509: $135-138$.

28 Tan C, Huang Y, Shi F, et al. C-reactive protein correlates with computed tomographic findings and predicts severe COVID-19 early. J Med Virol. 2020;92(7): 856-862.

29 Kong M, Zhang H, Cao X, Mao X, Lu Z. Higher level of neutrophil-to-lymphocyte is associated with severe COVID-19. Epidemiol Infect. 2020;148:e139.

30 Wang S, Fu L, Huang K, Han J, Zhang R, Fu Z. Neutrophil-to-lymphocyte ratio on admission is an independent risk factor for the severity and mortality in patients with coronavirus disease 2019. J Infect. 2020;82(2):e16-e18.

31 Cai J, Li H, Zhang C, et al. The neutrophil-to-lymphocyte ratio determines clinical efficacy of corticosteroid therapy in patients with COVID-19. Cell Metabol. 2021;33 (2):258-269. e253.

32 Fois AG, Paliogiannis P, Scano V, et al. The systemic inflammation index on admission predicts in-hospital mortality in COVID-19 patients. Molecules. 2020;25 (23).

33 Yang L, Liu S, Liu J, et al. COVID-19: immunopathogenesis and Immunotherapeutics. Signal Transduct Target Ther. 2020;5(1):128.

34 Barnes BJ, Adrover JM, Baxter-Stoltzfus A, et al. Targeting potential drivers of COVID-19: neutrophil extracellular traps. J Exp Med. 2020;217(6).

35 Laforge M, Elbim C, Frere C, et al. Tissue damage from neutrophil-induced oxidative stress in COVID-19. Nat Rev Immunol. 2020;20(9):515-516. 